\title{
Validation of Newly Proposed Time to Transarterial Chemoembolization Progression in Intermediate-Stage Hepatocellular Carcinoma Cases
}

\author{
Hirofumi Izumoto $^{a}$ Atsushi Hiraoka $^{a}$ Yoshihiro Ishimaru $^{\mathrm{b}}$ Tadashi Murakami $^{\mathrm{b}}$ \\ Shogo Kitahata ${ }^{a}$ Hidetaro Ueki ${ }^{a}$ Toshihiko Aibiki ${ }^{a}$ Tomonari Okudaira $^{a}$ \\ Yuji Miyamoto $^{a}$ Hiroka Yamago $^{a}$ Ryuichiro Iwasaki ${ }^{a}$ Hideomi Tomida $^{a}$ \\ Kenichiro Moria $^{a}$ Masato Kishida ${ }^{a}$ Eiji Tsubouchia Hideki Miyata ${ }^{a}$

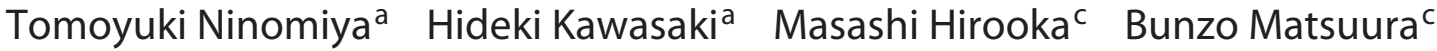 \\ Masanori Abe ${ }^{c}$ Yoichi Hiasa $^{c}$ Kojiro Michitaka ${ }^{a}$ Masatoshi Kudo ${ }^{d}$

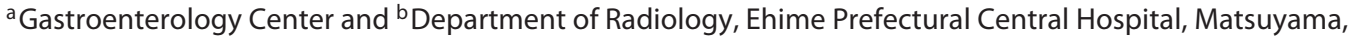 \\ 'Department of Gastroenterology and Metabology, Ehime University Graduate School of Medicine, Toon, and \\ ${ }^{\mathrm{d} D e p a r t m e n t}$ of Gastroenterology and Hepatology, Kindai University School of Medicine, Osaka-Sayama, Japan
}

\section{Keywords}

Hepatocellular carcinoma · Conventional transarterial chemoembolization - Barcelona Clinic Liver Cancer stage B · Transarterial chemoembolization-refractory status - Time to transarterial chemoembolization progression · Overall survival

\begin{abstract}
Background/Aim: Determination of failure of transarterial chemoembolization (TACE) for treatment of Barcelona Clinic Liver Cancer stage B (BCLC-B) hepatocellular carcinoma (HCC) has become important because of the development of tyrosine kinase inhibitor (TKI) treatment. We evaluated the usefulness and efficacy of the newly proposed time to TACE progression (TTTP). Patients and Methods: From 2006 to 2016, 192 BCLC-B HCC patients [median age 72 years,
\end{abstract}

\section{KARGER}

(C) 2017 S. Karger AG, Basel

E-Mail karger@karger.com

www.karger.com/ocl male/female ratio $=149 / 43$, Child-Pugh score 5/6/7 = 106/56/30, albumin-bilirubin (ALBI) grade 1/2 =64/128, Kinki criteria B1/B2 = 64/128] were enrolled. TTTP was defined based on a previous report and first imaging performed 3 months after initial TACE had been used to obtain baseline images. The patients were divided into three groups according to TTTP $(<5,5-10$, and $\geq 10$ months; group I, II, and III, respectively). We evaluated the relationship between TTTP and overall survival (OS) as well as the prognostic factors for death. Results: The median number of TACE procedures was 4 (interquartile range 3-7). There was a moderate correlation between TTTP and OS $(r=0.527,95 \% \mathrm{Cl} 0.416-$ $0.622, p<0.001)$. The median survival for group I $(n=78)$, II ( $n=49)$, and III ( $n=65)$ was $24.6,34.7$, and 49.5 months, respectively (group I vs. group II, $p=0.023$; group I vs. group III, $p<0.001$; group II vs. group III, $p=0.037$; Holm's method). ALBI grade 2 (HR 1.548, 95\% Cl 1.004-2.388, $p=0.048)$, al-

Dr. Atsushi Hiraok Gastroenterology Center Ehime Prefectural Central Hospital Kasuga-cho 83, Matsuyama, Ehime 790-0024 (Japan) E-Mail hirage@m.ehime-u.ac.jp 
pha-fetoprotein (>100 ng/mL) (HR 1.540, 95\% Cl 1.035$2.291, \mathrm{p}=0.033$ ), and TTTP ( $<5$ months) (HR $2.157,95 \% \mathrm{CI}$ $1.447-3.215, p<0.001$ ) were significant prognostic factors for death in multivariate Cox hazard analysis. Conclusion: In patients with reduced TTTP, especially $<5$ months, it might be difficult to improve prognosis with a repeated TACE procedures. In such cases, reconsideration of the therapeutic strategy might be needed when possible.

(c) 2017 S. Karger AG, Basel

\section{Introduction}

Hepatocellular carcinoma (HCC) is the fifth most common type of malignant tumor [1] and the third leading cause of cancer death throughout the world [2], though progress in the methods used for hepatic resection [3-6] and radiofrequency ablation [7-14] as curative treatments has improved the prognosis of affected patients. However, it is well known that recurrence of HCC frequently occurs following curative treatment. Transarterial chemoembolization (TACE) [15-19] has been developed as a palliative treatment for unresectable HCC in patients with or without a past history of treatments, especially for those in Barcelona Clinic Liver Cancer stage $\mathrm{B}$ (BCLC-B) (intermediate), and reported to be effective for improving patient prognosis.

Recently, systemic therapies with tyrosine kinase inhibitor (TKI) have been developed as treatments for unresectable HCC [20-26]. With recognition of the therapeutic effectiveness of TKI against HCC, the notion of TACE-refractory status has been proposed as an indication for switching from TACE to TKI [27], and Ogasawara et al. [28] and Arizumi et al. [19] reported the clinical importance of switching to sorafenib for intermediate-stage HCC patients to improve their prognosis.

The importance of determining TACE-refractory status and predicting progression in patients who have undergone repeated TACE procedures has increased, and as a new criterion for predicting poor prognosis, time to TACE progression (TTTP) has been proposed recently [29]. It has been shown that TTTP has a moderate correlation with overall survival (OS) after progression in patients with stage BCLC-B HCC who have been treated with TACE.

In the present study, we examined the usefulness of TTTP for predicting the prognosis in HCC patients with BCLC-B and good hepatic function (Child-Pugh score $\leq 7$ ) who underwent repeated TACE procedures.

\section{Patients and Methods}

\section{Patients}

From 2006 to 2016, 205 patients with Kinki criteria B1 or B2 substage HCC [30] were treated with TACE. After excluding 13 patients because of lack of follow-up imaging data for evaluation of TTTP, 192 were enrolled in this study.

\section{Diagnosis and Treatment of HCC}

HCC was diagnosed based on an increasing course of alphafetoprotein (AFP) as well as dynamic CT [31], MRI [32], contrastenhanced US with perfluorobutane (Sonazoid ${ }^{\circledR}$; Daiichi Sankyo Co., Ltd., Tokyo, Japan) $[33,34]$, and/or pathological findings. Tumor node metastasis (TNM) stage was determined using the criteria reported in studies for staging of HCC conducted by the Liver Cancer Study Group of Japan (LCSGJ, 6th edition) [35]. Prior to 2005, all enrolled patients were treated based on the HCC strategy developed by Ehime Prefecture Hospital. For those beyond the Milan criteria [36], surgical resection, if possible, was selected, while TACE was performed when surgical resection could not be performed [8]. Since 2005, all treatments were performed following the Japanese practical guidelines for HCC [37] whenever possible after obtaining written informed consent from each patient.

\section{Kinki Criteria for Substaging of BCLC-B HCC}

Using the reported Kinki criteria [30], patients were classified according to Child-Pugh score (5-7 or 8-9 points) and tumor burden (Milan criteria [36], up-to-seven criteria [38]). Staging for patients with a Child-Pugh score of 5-7 and within the up-to-seven criteria was B1, for those with a Child-Pugh score of 5-7 and beyond the up-to-seven criteria B2, and for those with a Child-Pugh score of 8-9 and any tumor status B3 [39].

\section{TACE Procedure}

All nodules treated with TACE were shown to be hypervascular. Two experienced radiologists (Y.I., T.M.) and a hepatologist (A.H.) performed the TACE procedures, for which a microcatheter was inserted into the artery feeding the tumor in a manner as superselective as possible following conventional hepatic angiography, after which a segmental or subsegmental TACE procedure was performed. Prior to the procedure, antegrade flow in the portal vein and absence of obstruction of the main trunk of the portal vein were confirmed by US, dynamic CT, and portography findings obtained via the superior mesenteric artery. Epirubicin hydrochloride (Farmorubicin ${ }^{\circledR}$; Pfizer Japan Inc., Tokyo, Japan), before January 2010, or miriplatin hydrate (MIRIPLA ${ }^{\circledR}$; Sumitomo Dainippon Pharma Co., Ltd., Osaka, Japan), after February 2010, was injected together with Lipiodol in all cases, after which a gelatin sponge cut into small fragments $\left(\right.$ Gelfoam $^{\circledR}$; Upjohn, Kalamazoo, MI, USA), before August 2006, or small gelatin sponge fragments (Gelpart ${ }^{\circledR}$; Nippon Kayaku Co., Ltd., Tokyo, Japan), after September 2006, were used for embolization. The goal of embolization was disappearance of tumor staining without complete obstruction of the hepatic artery. Three months after TACE, dynamic CT and/or EOB-MRI were performed. When intrahepatic recurrence was confirmed, the TACE procedure was repeated. 
Table 1. Clinical characteristics of 192 patients with BCLC-B hepatocellular carcinoma

\begin{tabular}{lc}
\hline Age, years & $72(64-77)$ \\
Gender (male/female) & $149 / 43$ \\
Etiology (HCV/HBV/HBV+HCV/ & \\
$\quad$ alcohol/others) & $149 / 5 / 1 / 11 / 26$ \\
Aspartate aminotransferase, IU/L & $52(35-79)$ \\
Alanine aminotransferase, IU/L & $39(25-65)$ \\
Platelets, $\times 10^{4}$ cells/ $\mu \mathrm{L}$ & $10.8(7.2-15.0)$ \\
Total bilirubin, mg/dL & $0.8(0.6-1.0)$ \\
Albumin, g/dL & $3.8(3.4-4.0)$ \\
Prothrombin time, \% & $81.5(73.9-91.0)$ \\
Child-Pugh class $(5 / 6 / 7)$ & $106 / 56 / 30$ \\
ALBI grade $(1 / 2)$ & $64 / 128$ \\
Alpha-fetoprotein, ng/mL & $30.1(9.1-160.325)$ \\
Fucosylated alpha-fetoprotein, $\%$ & $4.95(0.5-22.5)$ \\
PIVKA-II, mAU/mL & $85(29-594.5)$ \\
Maximum tumor size, cm & $1.9(1.2-3.4)$ \\
Tumor number $($ single/multiple) & $2 / 190$ \\
TNM stage of LCSGJ (II/III) & $104 / 88$ \\
Kinki criteria (B1/B2) & $64 / 128$ \\
Naïve/recurrence & $52 / 140$ \\
TTTP (<5/5-10/ $\geq 10$ months) & $78 / 49 / 65$
\end{tabular}

Values are presented as $n$ or median (interquartile range). ALBI, albumin-bilirubin; BCLC-B, Barcelona Clinic Liver Cancer stage $\mathrm{B}$; $\mathrm{HBV}$, hepatitis B virus; $\mathrm{HCV}$, hepatitis $\mathrm{C}$ virus; LCSGJ, Liver Cancer Study Group of Japan 6th edition; PIVKA-II, protein induced by vitamin $\mathrm{K}$ absence or antagonist-II; TNM, tumor node metastasis; TTTP, time to transarterial chemoembolization progression.

Time to TACE Progression

TTTP was defined based on findings previously reported [29]. For baseline imaging, the results of dynamic CT or EOB-MRI performed 3 months after the initial TACE were used, then findings from dynamic CT or EOB-MRI obtained 3 months after TACE were utilized for comparison to the baseline images. To assess the target lesions, the five largest tumors shown in the images were selected from intrahepatic lesions for calculating the total maximum diameter (two-dimensional measurement) of each viable lesion. When the total maximum diameter as compared to that seen in the baseline images was $\geq 1.2$ times larger, progression was determined.

When complete response was noted in the first imaging, the date of confirmed recurrence of HCC was defined as the date of progression, while the appearance of vascular invasion or extrahepatic spreading was also regarded as progression. Patients were divided into three groups based on TTTP $(<5$ months [group I], 5-10 months [group II], and $\geq 10$ months [group III]), then OS after progression was evaluated.

Written informed consent was obtained from all patients prior to treatment. The study protocol was in compliance with the Helsinki Declaration and approved by the Institutional Ethics Committee of Ehime Prefectural Central Hospital (No. 29-36).

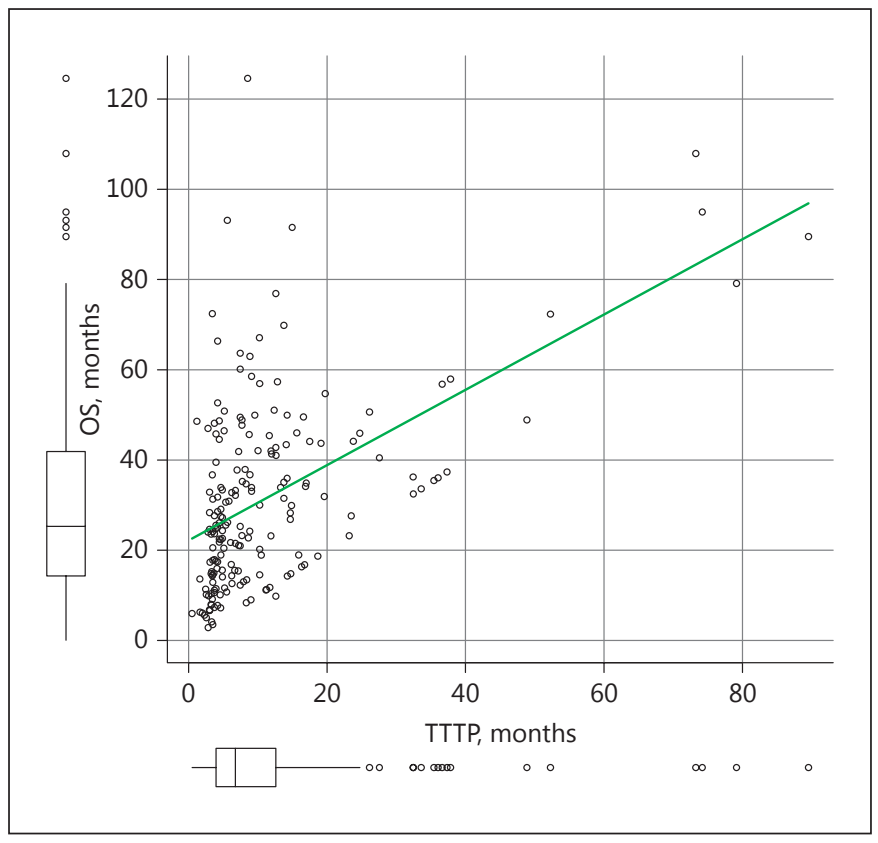

Fig. 1. Correlation between time to transarterial chemoembolization progression (TTTP) and overall survival (OS) after introducing transarterial chemoembolization (TACE). There was a moderate correlation between TTTP and OS after introducing TACE as treatment for Barcelona Clinic Liver Cancer stage B hepatocellular carcinoma patients $(r=0.527,95 \%$ CI $0.416-0.622, p<0.001)$.

\section{Statistical Analysis}

Statistical analyses were performed using the Kaplan-Meier method with log-rank test, and Pearson correlation test, Student $t$ test, Welch test, Mann-Whitney test, $\chi^{2}$ test, or Fisher exact test, as appropriate. Multiple comparisons were analyzed using Holm's method. Statistical significance was defined as $p<0.05$. Statistical analyses were performed using EZR, a graphical user interface for $\mathrm{R}$, version 1.32 (The R Foundation for Statistical Computing, Vienna, Austria).

\section{Results}

The clinical background information is shown in Table 1 . The median number of TACE procedures was 4 (interquartile range $3-7$ ). There was a modest correlation between TTTP and OS ( $r=0.527,95 \%$ CI 0.416-0.622, $p<0.001$ ) (Fig. 1).

The OS rate for each group based on TTTP was analyzed. Stratification ability using survival curves for each of those groups was good, with significant differences between them observed. The median survival time for group I $(n=78)$ was 24.6 months, while that of group II $(n=49)$ 
Table 2. Cox hazard analysis of prognostic factors for death after introduction of transarterial chemoembolization

\begin{tabular}{|c|c|c|c|c|c|c|}
\hline & \multicolumn{3}{|c|}{ Univariate } & \multicolumn{3}{|c|}{ Multivariate } \\
\hline & HR & $95 \% \mathrm{CI}$ & $p$ value & $\mathrm{HR}$ & $95 \% \mathrm{CI}$ & $p$ value \\
\hline Age ( $\geq 65$ years) & 1.139 & $0.754-1.72$ & 0.573 & - & - & - \\
\hline Gender (female) & 1.425 & $0.935-2.171$ & 0.100 & - & - & - \\
\hline Positive for hepatitis $C$ virus & 1.184 & $0.758-1.851$ & 0.458 & - & - & - \\
\hline Aspartate aminotransferase (>40 IU/L) & 1.636 & $1.104-2.423$ & 0.014 & 1.459 & $0.964-2.207$ & 0.074 \\
\hline Alanine aminotransferase (>40 IU/L) & 1.233 & $0.867-1.753$ & 0.244 & - & - & - \\
\hline Platelets $\left(>10 \times 10^{4}\right.$ cells $\left./ \mu \mathrm{L}\right)$ & 0.711 & $0.500-1.023$ & 0.059 & - & - & - \\
\hline Child-Pugh B (7) & 1.386 & $0.864-2.222$ & 0.176 & - & - & - \\
\hline Albumin-bilirubin (grade 2) & 1.887 & $1.265-2.814$ & 0.002 & 1.548 & $1.004-2.388$ & 0.048 \\
\hline Alpha-fetoprotein $(>100 \mathrm{ng} / \mathrm{mL})$ & 1.554 & $1.506-2.285$ & 0.025 & 1.540 & $1.035-2.291$ & 0.033 \\
\hline Fucosylated alpha-fetoprotein $(>10 \%)$ & 1.357 & $0.938-1.963$ & 0.105 & - & - & - \\
\hline PIVKA-II (>100 mAU/mL) & 1.488 & $1.041-2.127$ & 0.029 & 1.180 & $0.789-1.764$ & 0.421 \\
\hline Maximum tumor diameter $(>5 \mathrm{~cm})$ & 1.347 & $0.740-2.452$ & 0.330 & - & - & - \\
\hline TNM stage III of LCSGJ & 1.498 & $1.053-2.132$ & 0.025 & 1.084 & $0.735-1.600$ & 0.682 \\
\hline Kinki criteria B2 & 1.308 & $0.895-1.911$ & 0.165 & - & - & - \\
\hline Recurrence of hepatocellular carcinoma & 1.024 & $0.678-1.546$ & 0.910 & - & - & - \\
\hline TTTP (<5 months $)$ & 2.514 & $1.731-3.650$ & $<0.001$ & 2.157 & $1.447-3.215$ & $<0.001$ \\
\hline
\end{tabular}

LCSGJ, Liver Cancer Study Group of Japan 6th edition; PIVKA-II, protein induced by vitamin K absence or antagonist-II; TNM, tumor node metastasis; TTTP, time to transarterial chemoembolization progression.

was 34.7 months and that of group III $(n=65) 49.5$ months (group I vs. group II, $p=0.023$; group I vs. group III, $p<0.001$; group II vs. group III, $p=0.037$, respectively; Holm's method) (Fig. 2).

In univariate Cox hazard analysis, aspartate aminotransferase ( $>40 \mathrm{IU} / \mathrm{L}$ ), albumin-bilirubin (ALBI) grade 2 , AFP $(>100 \mathrm{ng} / \mathrm{mL})$, protein induced by vitamin $\mathrm{K}$ absence or antagonist-II (PIVKA-II) (>100 mAU/mL), TNM stage III, and TTTP ( $<5$ months) were significant prognostic factors for death after TACE introduction for BCLC-B HCC. Multivariate analysis showed that ALBI grade 2 (HR 1.548, 95\% CI 1.004-2.388, $p=0.048$ ), AFP $(>100 \mathrm{ng} / \mathrm{mL})(\mathrm{HR} 1.540,95 \%$ CI $1.035-2.291, p=0.033)$, and TTTP ( $<5$ months) (HR 2.157, 95\% CI 1.447-3.215, $p<0.001$ ) were significant prognostic factors (Table 2).

In a comparison between Kinki criteria B1 $(n=64)$ and B2 $(n=128)$ cases, Child-Pugh score/classification was not significantly different $(p=0.818$ ), while the frequency of TNM stage III was greater in the B2 subgroup ( $p=$ 0.001 ) (data not shown). Although the survival curve for the $\mathrm{B} 1$ cases was superior to that of the B2 cases, no significant difference was seen (median survival time: B1 vs. B2 $=44.1$ vs. 34.1 months, $p=0.161$ ). Furthermore, no significant difference was observed between B1 and B2 with regard to TTTP (median TTTP: B1 vs. B2 $=7.2$ vs. $6.8, p=0.899$ ).

Time to TACE Progression with Repeated TACE

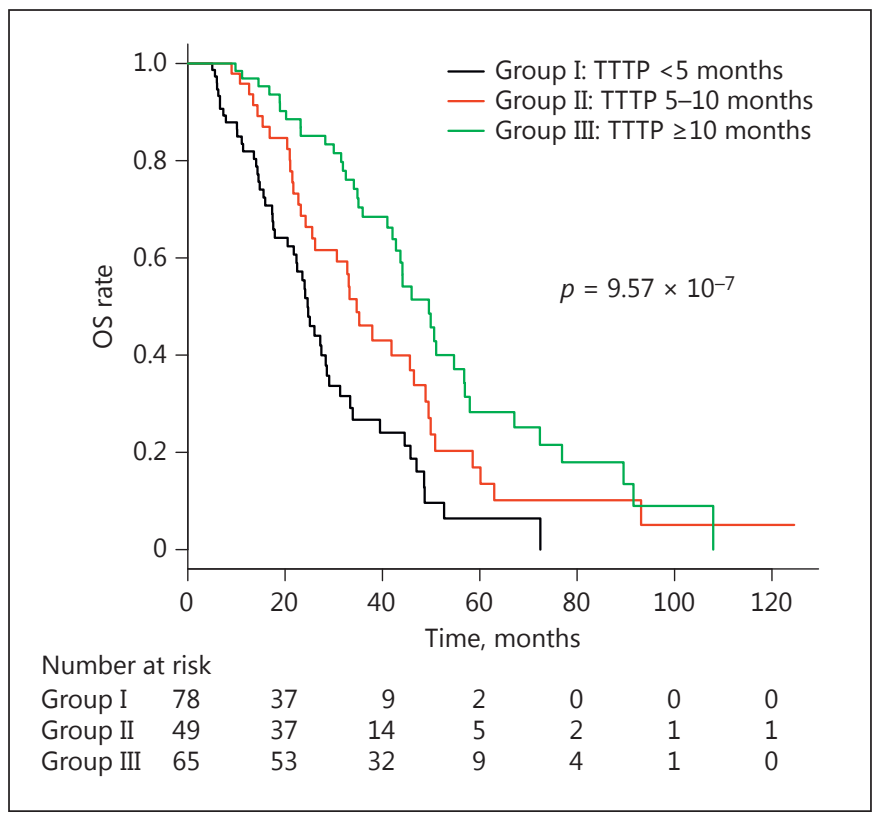

Fig. 2. Overall survival (OS) rates in each group based on time to transarterial chemoembolization progression (TTTP) grouping. Significant differences were observed among the OS rate values for all groups. The median survival time for group I ( $n=78$, TTTP $<5$ months) was 24.6 months, for group II ( $n=49$, TTTP $5-10$ months) 34.7 months, and for group III ( $n=65$, TTTP $\geq 10$ months $) 49.5$ months (group I vs. group II, $p=0.023$; group I vs. group III, $p<$ 0.001 ; group II vs. group III, $p=0.037$, respectively; Holm's method).

Oncology 2017;93(suppl 1):120-126 DOI: $10.1159 / 000481242$ 


\section{Discussion}

TACE-refractory status has been proposed in studies conducted in both Western and Asian countries [27, 40, 41]. Switching to TKI is considered important for improvement of prognosis in patients with BCLC-B HCC who are confirmed to be TACE refractory $[19,28]$. The usefulness of sorafenib for advanced HCC was demonstrated in the SHARP [20] and Asia-Pacific [42] trials. Moreover, regorafenib has been proposed for use as a second-line agent in cases receiving sorafenib therapy [21], while the effectiveness of lenvatinib as another first-line TKI agent for unresectable HCC has recently been reported $[22,23]$. In the future, determination of TACE-refractory status will increase in importance along with the introduction of therapeutic options for improvement of prognosis in HCC patients because of the increasing number of effective TKI treatments presently under development.

TTTP has been proposed as a new factor for determining progression in patients undergoing repeated TACE treatments. From the viewpoint of therapeutic response to TACE, TTTP showed a moderate correlation to OS after introduction of TACE, and OS was reduced in the patients with shorter TTTP (group I) as compared to the others. These results were similar to those of the previous report that originally proposed TTTP [29].

In addition, maintained hepatic function is important for improving the prognosis of BCLC-B HCC patients, except for therapeutic response to repeated TACE procedures. In our previous study, hepatic function worsened with repeated TACE procedures [43], as determined with the use of the newly proposed ALBI score [44-46]. Some reports have noted the usefulness of ALBI grade for patients undergoing sorafenib treatment and for subclassification of BCLC-B HCC [47-49]. In fact, ALBI grade 2, but not Child-Pugh class B (score 7), was shown to be an independent risk factor for death by Cox hazard analysis findings in the present study. Since TKI therapy is recommended for patients with good hepatic function, it is important to keep in mind that noneffective TACE can also harm hepatic function, thus possibly reducing the opportunity for TKI treatment in the future.

Although the OS of the Kinki criteria B1 subgroup was not superior to that of the B2 subgroup in our study, previous reports with larger cohorts of patients classified as BCLC-B [30] according to the Kinki and Bolondi [49] criteria, used with Child-Pugh score, or the MICAN criteria [48], used with ALBI grade, have found that a lower subgrade is related to better prognosis. In our results, TTTP did not show significant differences when compared between the Kinki criteria B1 and B2 subgroups ( $p=0.899)$. The response to TACE might be similar for each subgrade of BCLC-B. For BCLC-B HCC patients treated with TACE in this multiple TKI era, prolonging postprogression survival is thought to be necessary for improving OS, thus identification of patients who easily develop TACE failure or refractory status has become important [50]. TTTP may be suitable for determining the optimal timing for switching to TKI while avoiding worsened hepatic function.

Our study has some limitations. First, this was a validation study based on retrospective analyses. Furthermore, all analyzed patients were treated at the same institution. Finally, a suitable assessment method for hepatic function with regard to TKI treatment remains to be established. In the future, a prospective study with a larger cohort treated at multiple institutions will be needed.

In conclusion, TTTP may be a good surrogate endpoint for use in a TACE combination trial with TKI or immunotherapy, because TTTP correlates with OS. In patients with reduced TTTP, especially $<5$ months, it might be difficult to improve prognosis by repeated TACE procedures. In such cases, reconsideration of the therapeutic strategy, including switching treatment from TACE to TKI, may be necessary.

\section{Disclosure Statement}

None of the authors has any financial disclosures, grants from any organs, or conflicts of interest to declare.

\footnotetext{
References

1 Parkin DM, Bray F, Ferlay J, Pisani P: Estimating the world cancer burden: Globocan 2000. Int J Cancer 2001;94:153-156.

2 Parkin DM, Bray F, Ferlay J, Pisani P: Global cancer statistics, 2002. CA Cancer J Clin 2005; 55:74-108.

3 Song TJ, Ip EW, Fong Y: Hepatocellular carcinoma: current surgical management. Gastroenterology 2004;127(5 suppl 1):S248-S260.

4 Seyama Y, Kokudo N: Assessment of liver function for safe hepatic resection. Hepatol Res 2009;39:107-116.

5 Kudo M, Izumi N, Sakamoto M, Matsuyama Y, Ichida T, Nakashima O, Matsui O, et al: Survival analysis over 28 years of $173,378 \mathrm{pa}-$ tients with hepatocellular carcinoma in Japan. Liver Cancer 2016;5:190-197.
}

Izumoto et al. 
6 Ho MC, Hasegawa K, Chen XP, Nagano H, Lee YJ, Chau GY, Zhou J, et al: Surgery for intermediate and advanced hepatocellular carcinoma: a consensus report from the 5th Asia-Pacific Primary Liver Cancer Expert Meeting (APPLE 2014). Liver Cancer 2016;5: 245-256.

7 Tateishi R, Shiina S, Teratani T, Obi S, Sato S, Koike Y, Fujishima T, et al: Percutaneous radiofrequency ablation for hepatocellular carcinoma. An analysis of 1000 cases. Cancer 2005;103:1201-1209.

8 Hiraoka A, Horiike N, Yamashita Y, Koizumi Y, Doi K, Yamamoto Y, Hasebe A, et al: Efficacy of radiofrequency ablation therapy compared to surgical resection in 164 patients in Japan with single hepatocellular carcinoma smaller than $3 \mathrm{~cm}$, along with report of complications. Hepatogastroenterology 2008; 55 : 2171-2174.

9 Hiraoka A, Michitaka K, Horiike N, Hidaka S, Uehara T, Ichikawa S, Hasebe A, et al: Radiofrequency ablation therapy for hepatocellular carcinoma in elderly patients. J Gastroenterol Hepatol 2010;25:403-407.

10 Kudo M: Surveillance, diagnosis, treatment, and outcome of liver cancer in Japan. Liver Cancer 2015;4:39-50.

11 Poon RT, Cheung TT, Kwok PC, Lee AS, Li TW, Loke KL, Chan SL, et al: Hong Kong consensus recommendations on the management of hepatocellular carcinoma. Liver Cancer 2015;4:51-69.

12 Kudo M: Clinical practice guidelines for hepatocellular carcinoma differ between Japan, United States, and Europe. Liver Cancer 2015; 4:85-95.

13 Kang TW, Rhim H: Recent advances in tumor ablation for hepatocellular carcinoma. Liver Cancer 2015;4:176-187.

14 Lencioni R, de Baere T, Martin RC, Nutting CW, Narayanan G: Image-guided ablation of malignant liver tumors: recommendations for clinical validation of novel thermal and non-thermal technologies - a Western perspective. Liver Cancer 2015;4:208-214.

15 Takayasu K, Arii S, Ikai I, Omata M, Okita K, Ichida T, Matsuyama Y, et al: Prospective cohort study of transarterial chemoembolization for unresectable hepatocellular carcinoma in 8510 patients. Gastroenterology 2006; 131:461-469.

16 Hiraoka A, Kumagi T, Hirooka M, Uehara T, Kurose K, Iuchi H, Hiasa Y, et al: Prognosis following transcatheter arterial embolization for 121 patients with unresectable hepatocellular carcinoma with or without a history of treatment. World J Gastroenterol 2006;12: 2075-2079.

17 Kudo M: Locoregional therapy for hepatocellular carcinoma. Liver Cancer 2015;4:163164.

18 Tsurusaki M, Murakami T: Surgical and locoregional therapy of HCC: TACE. Liver Cancer 2015;4:165-175.
19 Arizumi T, Ueshima K, Minami T, Kono M, Chishina H, Takita M, Kitai S, et al: Effectiveness of sorafenib in patients with transcatheter arterial chemoembolization (TACE) refractory and intermediate-stage hepatocellular carcinoma. Liver Cancer 2015;4:253-262.

20 Llovet JM, Ricci S, Mazzaferro V, Hilgard P, Gane E, Blanc JF, de Oliveira AC, et al: Sorafenib in advanced hepatocellular carcinoma. N Engl J Med 2008;359:378-390.

21 Bruix J, Qin S, Merle P, Granito A, Huang YH, Bodoky G, Pracht M, et al: Regorafenib for patients with hepatocellular carcinoma who progressed on sorafenib treatment (RESORCE): a randomised, double-blind, placebo-controlled, phase 3 trial. Lancet 2017; 389:56-66.

22 Ikeda K, Kudo M, Kawazoe S, Osaki Y, Ikeda M, Okusaka T, Tamai T, et al: Phase 2 study of lenvatinib in patients with advanced hepatocellular carcinoma. J Gastroenterol 2017; 52:512-519.

23 Cheng AL, Finn RS, Qin S, Han KH, Ikeda K, Piscaglia F, Baron AD, et al: Phase III trial of lenvatinib (LEN) vs sorafenib (SOR) in firstline treatment of patients (pts) with unresectable hepatocellular carcinoma (uHCC) (abstract). J Clin Oncol 2017;35:4001.

24 Kudo M: Molecular targeted therapy for hepatocellular carcinoma: where are we now? Liver Cancer 2015;4:i-vii.

25 Kudo M: Regorafenib as second-line systemic therapy may change the treatment strategy and management paradigm for hepatocellular carcinoma. Liver Cancer 2016;5:235-244.

26 Kudo M: Immune checkpoint blockade in hepatocellular carcinoma. Liver Cancer 2015;4: 201-207.

27 Kudo M, Matsui O, Izumi N, Kadoya M, Okusaka T, Miyayama S, Yamakado K, et al: Transarterial chemoembolization failure/refractoriness: JSH-LCSGJ criteria 2014 update. Oncology 2014;87(suppl 1):22-31.

28 Ogasawara S, Chiba T, Ooka Y, Kanogawa N, Motoyama T, Suzuki E, Tawada A, et al: Efficacy of sorafenib in intermediate-stage hepatocellular carcinoma patients refractory to transarterial chemoembolization. Oncology 2014;87:330-341.

29 Arizumi T, Ueshima K, Iwanishi M, Minami T, Chishina H, Kono M, Takita M, et al: The overall survival of patients with hepatocellular carcinoma correlates with the newly defined time to progression after transarterial chemoembolization. Liver Cancer 2017;6: 227-235.

30 Kudo M, Arizumi T, Ueshima K, Sakurai T, Kitano M, Nishida N: Subclassification of BCLC B stage hepatocellular carcinoma and treatment strategies: proposal of modified Bolondi's subclassification (Kinki criteria). Dig Dis 2015;33:751-758.
31 Bruix J, Sherman M: Management of hepatocellular carcinoma. Hepatology 2005;42: 1208-1236.

32 Sano K, Ichikawa T, Motosugi U, Sou H, Muhi AM, Matsuda M, Nakano M, et al: Imaging study of early hepatocellular carcinoma: usefulness of gadoxetic acid-enhanced MR imaging. Radiology 2011;261:834-844.

33 Hiraoka A, Hiasa Y, Onji M, Michitaka K: New contrast enhanced ultrasonography agent: impact of Sonazoid on radiofrequency ablation. J Gastroenterol Hepatol 2011;26: 616-618.

34 Kudo M: Breakthrough imaging in hepatocellular carcinoma. Liver Cancer 2016;5:47-54.

35 The Liver Cancer Study Group of Japan Society of Hepatology: The General Rules for the Clinical and Pathological Study of Primary Liver Cancer, ed 6. Tokyo, Kanehara \& Co Ltd, 2015, p 26.

36 Mazzaferro V, Regalia E, Doci R, Andreola S, Pulvirenti A, Bozzetti F, Montalto F, et al: Liver transplantation for the treatment of small hepatocellular carcinomas in patients with cirrhosis. N Engl J Med 1996;334:693-699.

37 The Japan Society of Hepatology: Clinical Practice Guidelines for Hepatocellular Carcinoma 2013. https://www.jsh.or.jp/English/ guidelines_en/Guidelines_for_hepatocellular_carcinoma_2013 (accessed August 1, 2017).

38 D'Amico F, Schwartz M, Vitale A, Tabrizian $\mathrm{P}$, Roayaie S, Thung S, Guido M, et al: Predicting recurrence after liver transplantation in patients with hepatocellular carcinoma exceeding the up-to-seven criteria. Liver Transpl 2009;15:1278-1287.

39 Kudo M: Heterogeneity and subclassification of Barcelona Clinic Liver Cancer Stage B. Liver Cancer 2016;5:91-96.

40 Hucke F, Sieghart W, Pinter M, Graziadei I, Vogel W, Muller C, Heinzl H, et al: The ARTstrategy: sequential assessment of the ART score predicts outcome of patients with hepatocellular carcinoma re-treated with TACE. J Hepatol 2014;60:118-126.

41 Dufour JF, Bargellini I, De Maria N, De Simone P, Goulis I, Marinho RT: Intermediate hepatocellular carcinoma: current treatments and future perspectives. Ann Oncol 2013; 24(suppl 2):ii24-ii29.

42 Cheng AL, Kang YK, Chen Z, Tsao CJ, Qin S, Kim JS, Luo R, et al: Efficacy and safety of sorafenib in patients in the Asia-Pacific region with advanced hepatocellular carcinoma: a phase III randomised, double-blind, placebo-controlled trial. Lancet Oncol 2009; 10:25-34.

43 Hiraoka A, Kumada T, Kudo M, Hirooka M, Koizumi Y, Hiasa Y, Tajiri K, et al: Hepatic function during repeated TACE procedures and prognosis after introducing sorafenib in patients with unresectable HCC - multicenter analysis. Dig Dis 2017;35:602-610. 
44 Johnson PJ, Berhane S, Kagebayashi C, Satomura S, Teng M, Reeves HL, O'Beirne J, et al: Assessment of liver function in patients with hepatocellular carcinoma: a new evidencebased approach - the ALBI grade. J Clin Oncol 2015;33:550-558.

45 Hiraoka A, Kumada T, Michitaka K, Toyoda H, Tada T, Ueki H, Kaneto M, et al: Usefulness of albumin-bilirubin grade for evaluation of prognosis of 2584 Japanese patients with hepatocellular carcinoma. J Gastroenterol Hepatol 2016;31:1031-1036.
46 Hiraoka A, Kumada T, Kudo M, Hirooka M, Tsuji K, Itobayashi E, Kariyama K, et al: Albumin-bilirubin (ALBI) grade as part of the evidence-based clinical practice guideline for HCC of the Japan Society of Hepatology: a comparison with the liver damage and ChildPugh classifications. Liver Cancer 2017;6: 204-215.

47 Ogasawara S, Chiba T, Ooka Y, Suzuki E, Kanogawa N, Saito T, Motoyama T, et al: Liver function assessment according to the albumin-bilirubin (ALBI) grade in sorafenibtreated patients with advanced hepatocellular carcinoma. Invest New Drugs 2015;33:12571262.
48 Hiraoka A, Kumada T, Nouso K, Tsuji K, Itobayashi E, Hirooka M, Kariyama K, et al: Proposed new sub-grouping for intermediatestage hepatocellular carcinoma using albumin-bilirubin grade. Oncology 2016;91: 153-161.

49 Bolondi L, Burroughs A, Dufour JF, Galle PR, Mazzaferro V, Piscaglia F, Raoul JL, et al: Heterogeneity of patients with intermediate (BCLC B) hepatocellular carcinoma: proposal for a subclassification to facilitate treatment decisions. Semin Liver Dis 2012;32:348-359.

50 Kudo M: A new era of systemic therapy for hepatocellular carcinoma with regorafenib and lenvatinib. Liver Cancer 2017;6:177-184. 